\title{
Analysis of 85 Cases of Minimal Media Lower Hemisternotomy for Congenital Cardiac Surgery Under Cardiopulmonary Bypass in Infants
}

\author{
Yuehu Han, ${ }^{1}$ Kexun Chen, ${ }^{2}$ Zhifa Wang, ${ }^{1}$ Dongming Wei, ${ }^{1}$ Yanjie Guo, ${ }^{3}$ Le Duan, ${ }^{1}$ Jie Su,,${ }^{1}$ Tao Chen ${ }^{1}$, \\ Chunhu $\mathrm{Gu}^{1}$ \\ ${ }^{1}$ Department of Cardiovascular Surgery, Xijing Hospital, Fourth Military Medical University, Xi'an, China; ${ }^{2}$ Department of \\ rehabilitation medicine, Shenzhen hospital of Southern medical university, Shenzhen, China; ${ }^{3}$ Department of Cardiology, Xijing \\ Hospital, Fourth Military Medical University, Xi'an, China
}

\section{ABSTRACT}

Objective: To investigate the feasibility and effect of minimal media lower hemisternotomy for cardiac surgery under cardiopulmonary bypass (CPB) in infant congenital heart disease.

Methods: In our hospital from May 2019 to October 2019, 170 infants with congenital heart disease underwent surgical treatment (median age 6.6 months; weight $6.0 \mathrm{~kg}$ ). They were divided into 2 groups: those with conventional chest median incision and those with minimal sternotomy. Minimal lower hemisternotomy began from the third intercostal level and ended $0.5 \mathrm{~cm}$ above the xiphoid, just enough to insert a small sternal distractor.

Results: There was no significant difference between the 2 groups in CPB time. The operation time of small incision group was slightly longer $(\mathrm{P}<.05)$. There was no difference in prognosis between the 2 groups, but the wound length of the small incision group was significantly reduced $(4.0 \pm 0.5$ versus $7.8 \pm 0.8 \mathrm{~cm}, \mathrm{P}<.05)$. Time of intensive care unit and hospital stay was shorter among hemisternotomy patients at a statistically significant level $(\mathrm{P}<.05)$.

Conclusion: Minimal media lower hemisternotomy with the basic advantages of the sternal incision can expose the various parts of the heart, which meets most cardiac exploration and surgical operation needs, and the incision may still be extended if necessary. Lower hemisternotomy appears to be a safe, effective, and versatile alternative for many surgical interventions in infants with congenital heart disease.

Received fanuary 2, 2021; received in revised form fanuary 30, 2021; accepted February 1, 2021.

Y. Han, K. Chen, Z. Wang, D. Wei, and Y. Guo contributed equally to this work.

Correspondence: Fie Su, Department of Cardiovascular Surgery, Xijing Hospital, Fourth Military Medical University, Xi'an, China; 15809281217 (e-mail: 15809281217@163.com); Tao Chen, Department of Cardiovascular Surgery, Xijing Hospital, Fourth Military Medical University, Xi'an, China; 18629287449 (e-mail: felix85212@163.com); Chunbu Gu, Department of Cardiovascular Surgery, Xijing Hospital, Fourth Military Medical University, Xi'an, China; 18629267464 (e-mail: 84733952@qq.com).

\section{INTRODUCTION}

The standard approach for congenital cardiac disease surgery is the median sternotomy, which offers unrestricted access to the thoracic organs: heart, lung, and major vessels. The mortality and morbidity rate in repairing some congenital heart disease (CHD) defects with median sternotomy procedure is extremely low, including atrial septal defects (ASDs), ventricular septal defects (VSDs), and patent ductus arteriosus. However, developments in interventional cardiology have made the repair of some CHDs with transcatheterization a superior alternative to surgery because it is minimally invasive and has improved cosmetic outcome. Minimally invasive cardiac surgery has evolved rapidly over the last decade because of the cosmetics and some psychological results connected to it [Neves 2018; Ha 2018; Fleißner 2019; Hata 2018]. These days, for ASD or VSD closure in young women and children, one such minimal invasive procedure is mini-sternotomy for right anterior thoracotomy, right anterolateral thoracotomy, or posterior thoracotomy. The purpose of our study is to compare the feasibility and effect of minimal media lower hemisternotomy to conventional full sternotomy for cardiac surgery under $\mathrm{CPB}$ in infants with CHD.

\section{METHODS}

\section{Patients}

This retrospective study included 170 CHD patients who underwent surgery in our department between May 2019 and October 2019. The patients were divided into 2 groups according to the surgical incision and technique used. The trial was approved by the Institutional Ethics Committee of our center. Before undergoing either conventional full sternotomy or minimal lower hemisternotomy, the patients' parents were made aware of the differences between the 2 procedures.

\section{Surgical Technique}

The standard media sternotomy is not described in detail here; we mainly introduce the lower hemisternotomy technique. A skin incision is created from the third interspace 

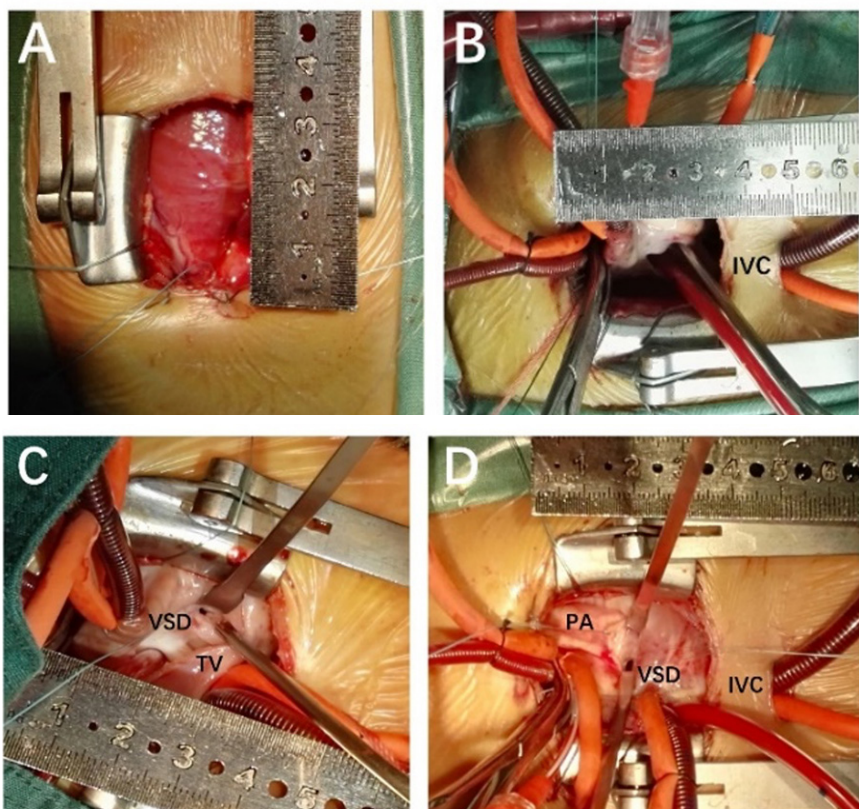

Figure 1. Surgery pictures. A and B, Lower hemisternotomy incision. C, Ventricular septal defect (perimembranous). D, Ventricular septal defect (supracristal). IVC, inferior vena cava; PA, pulmonary artery; TV, tricuspid valve.

extending inferiorly to $0.5 \mathrm{~cm}$ from the xiphoid, resulting in an approximately $4 \mathrm{~cm}$ incision. Diathermy dissection through the subcutaneous tissues defines the midline of the sternum. The hemisternotomy is commenced from the xiphoid to the manubriosternal junction using a handheld sternal saw. Time spent on hemostasis at this point is essential; bleeding edges of the periosteum are managed with diathermy, and hemostasis of the cut surface of the sternum is achieved using a small amount of bone wax. The pericardium is opened superiorly from the pericardial reflection. Standard purse sutures and $\mathrm{CPB}$ cannulation are made. Because of the short incision, the inferior vena cava cannula moving on the incision area can create difficulties in manipulation as the operation progresses. Therefore, a small skin incision is made in the lower xiphoid, which is used for drainage of the inferior vena cava during the operation and as a subcutaneous drain postoperatively (Figure 1). Thus, better exposure is provided in the surgical field during the operation, and the site where the cannula passed is used as the thorax drain after the surgery. In addition, we have designed a special surgical retractor to aid in the operation that provides better exposure compared with conventional retractors (patent applied) (Figure 2). The surgery technique of intracardiac malformation correction is similar to that used in conventional sternotomy.

\section{Statistical Analysis}

SPSS for Windows version 12.0 was used for statistical analysis. Quantitative data are given as mean \pm standard deviation. In the comparison of normal variables between the 2 groups, we used the independent sample t test. All tests were mutual, and $\mathrm{P}<.05$ was considered statistically significant.
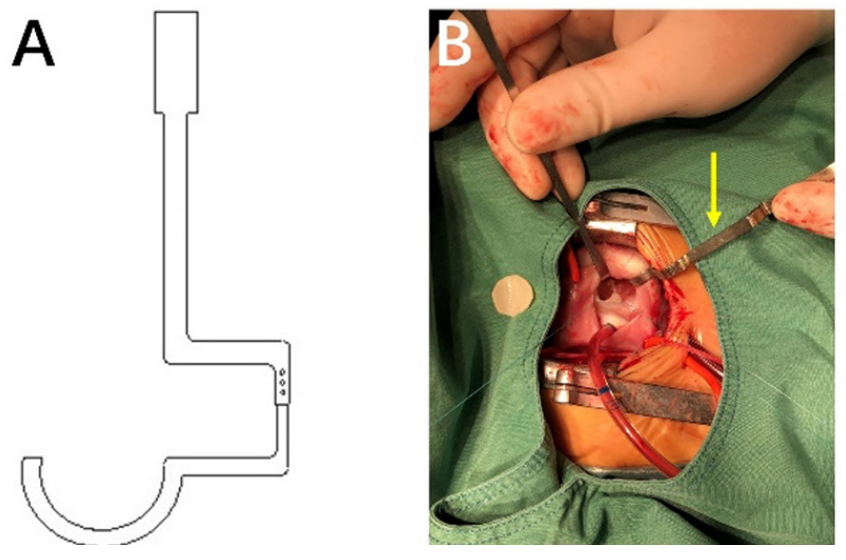

Figure 2. Special surgical retractor to match the lower hemisternotomy incision procedure provides better exposure compared with the conventional one by getting around the chest wall.

\section{RESULTS}

\section{Study Populations}

The preoperative demographic and diagnostic data of the study groups are detailed in Table 1 . There were 85 male and 85 female patients. The age ranged from 3 to 17 months (average 6.9). The weight ranged from 3.5 to $11 \mathrm{~kg}$ (average 6.9). The diagnoses included ventricular septal defects (VSDs), total anomalous pulmonary venous connection and tetralogy of Fallot (TOF).

\section{Operation Data}

The results obtained from the operations are given in Table 2. It is statistically significant that the length of incision was clearly shorter in the hemisternotomy group than in the standard sternotomy group, and that the operation time was longer in the hemisternotomy group than in the standard sternotomy group $(\mathrm{P}<.05)$. No statistical difference was observed between the 2 groups in terms of CBP time, aortic cross-clamp (ACC) time, or bleeding amount during surgery.

\section{Clinical Outcomes}

In the postoperative period, time of intensive care unit (ICU) and hospital stay was shorter among hemisternotomy patients at a statistically significant level $(\mathrm{P}<.05)$. The average follow-up period was 24 months.

\section{DISCUSSION}

The median thoracic incision is generally recognized as the best and safest option for cardiac surgery. It is basically applicable to all kinds of cardiac malformations. However, the huge surgical scars and disposal of surrounding tissues (children's thymus) are drawbacks. With the development of surgical instruments and increasing aesthetic requirements, minimally invasive open heart surgery has attracted more and more attention from patients and surgeons [Reardon 1999]. In addition to the median incision, there are several types of 
Table 1. Clinical Data of CHD Patients*

\begin{tabular}{lcc}
\hline Characteristic & $\begin{array}{c}\text { Full Sternotomy } \\
(\mathrm{n}=85)\end{array}$ & $\begin{array}{c}\text { Lower } \\
(\mathrm{n}=85)\end{array}$ \\
\hline Male & 43 & 42 \\
Female & 42 & 43 \\
Age (mo) & $6.4 \pm 1.8$ & $6.9 \pm 2.1$ \\
Weight (kg) & $5.9 \pm 2.1$ & $6.2 \pm 1.9$ \\
Cardiothoracic ratio & $0.59 \pm 0.1$ & $0.61 \pm 0.1$ \\
LVEF, preoperative (\%) & $60.5 \pm 2.5$ & $61.2 \pm 2.6$ \\
VSD (perimembranous) & 65 & 67 \\
VSD (supracristal) & 9 & 8 \\
VSD (muscular) & 4 & 5 \\
TAPVC (intracardiac) & 3 & 2 \\
TOF & 4 & 3 \\
\hline
\end{tabular}

*Data are $\mathrm{n}$ or mean \pm standard deviation.

LVEF indicates left ventricular ejection fraction; TAPVC, total anomalous

pulmonary venous connection.

surgical approach for cardiac diseases. Although these incisions reduce scars and avoid sternotomy, they cannot provide the surgical vision, operation space, and safety of a conventional median sternotomy incision. In addition, because of infants' age, weight, body shape, and peripheral vascular development, minimally invasive cardiac surgery for infants is less than optimal.

The hemisternotomy compared with the standard midline full sternotomy incision has been suggested as a minimally invasive technique, potentially maintaining better sternal stability and limiting postoperative sternal wound complications. Several groups have reported the feasibility and safety of hemisternotomy, and it has become a well-accepted alternative for patients undergoing aortic valve procedures [Kim 2006]. However, the hemisternotomy is often not considered for a variety of other cardiac surgical interventions, such as CHD for infants. Nevertheless, the reduced incision provides excellent exposure for numerous cardiac procedures and allows for standard $\mathrm{CPB}$ cannulation and surgical equipment. The hemisternotomy is also advantageous over other minimally invasive procedures as it can quickly and easily be extended into a full midline sternotomy for maximum exposure in the event of complications.

Considering the cosmetic and psychological needs of patients, we have performed lower hemisternotomy surgery for infants with CHD. We demonstrate that many cardiac procedures for infants can be performed via a lower hemisternotomy incision using conventional techniques. These procedures include septal defects, total anomalous pulmonary venous drainage, and TOF. Previous studies have suggested that the hemisternotomy could be beneficial in areas such as incisional pain, length of stay, cosmetics, sternal stability, and overall cost [Cohn 1997; Corbi 2003; Bakir 2006].
Table 2. Operative and Postoperative Data*

\begin{tabular}{lcc}
\hline Factor & $\begin{array}{c}\text { Full Sternotomy } \\
(\mathrm{n}=85)\end{array}$ & $\begin{array}{c}\text { Lower Hemisternotomy } \\
(\mathrm{n}=85)\end{array}$ \\
\hline Incision length (cm) & $7.8 \pm 0.8$ & $4.0 \pm 0.5 \dagger$ \\
Operation time (min) & $81.6 \pm 3.8$ & $95.2 \pm 2.0 \dagger$ \\
CPB time (min) & $58.6 \pm 18.1$ & $60.5 \pm 14.5$ \\
ACC time (min) & $25.5 \pm 12.2$ & $27.6 \pm 11.5$ \\
Blood volume (mL) & $35.5 \pm 10.8$ & $37.6 \pm 13.2$ \\
Ventilation time (h) & $18.6 \pm 3.9$ & $14.2 \pm 2.0$ \\
ICU stay (h) & $30.5 \pm 5.2$ & $22.6 \pm 3.6 \dagger$ \\
Hospital stay (d) & $9 \pm 4$ & $8 \pm 4 \dagger$ \\
Poor wound healing & $3(3.5)$ & $2(2.4)$ \\
Nosocomial infection & $5(5.9)$ & $6(7.1)$ \\
Mortality & 0 & 0 \\
\hline
\end{tabular}

*Data are mean \pm standard deviation or $\mathrm{n}(\%)$. $\dagger P<.05$

We found that the operation times of the small incision group were slightly longer $(\mathrm{P}<.05)$. $\mathrm{CPB}$ and clamp times were found to be comparable between the 2 groups, suggesting that despite the reduced incision, the hemisternotomy provides adequate visualization for procedures to be carried out in a timely manner. Lower hemisternotomy patients tended to spend less time in the intensive care unit and hospital postoperatively.

In conclusion, we have demonstrated the versatility of the lower hemisternotomy incision for CHD of infants and its safety compared with the conventional approach. Despite reports that cosmesis is of low priority for many patients, everincreasing patient awareness and available information will lead to increased requests. Cardiac surgeons could be familiar with this technique and strive for new ways to test its value compared with the gold standard, the complete sternotomy.

\section{ACKNOWLEDGMENTS}

This work was supported by grants from the Natural Science Foundation of Shaanxi Province (2019JQ575), the Boost Foundation (XJZT18MJ48, XJZT19Z12), and National Key Research and Development Program (2017YFC1308101, 2018YFC1002603).

\section{REFERENCES}

Bakir I, Casselman F, Wellens F, et al. Minimally invasive versus standard approach aortic valve replacement: A study in 506 patients. Ann Thorac Surg 2006;81:1599-1604.

Cohn LH, Adams DH, Couper GS, et al. Minimally invasive cardiac valve surgery improves patient satisfaction while reducing costs of cardiac valve replacement and repair. Ann Surg 1997;226:421-428. 
Corbi P, Rahmati M, Donal E, et al. Prospective comparison of minimally invasive and standard techniques for aortic valve replacement: Initial experience in the first hundred patients. J Card Surg 2003;18:133-139.

Fleißner F, Salman J, Naqizadah J, Avsar M. Minimally invasive surgery in mitral valve endocarditis. Thorac Cardiovasc Surg 2019;67:637-643.

Ha KJ, Cho WC, Kim WK, Kim JB. A minimally invasive approach for the treatment of mid-aortic syndrome in Takayasu arteritis. Korean J Thorac Cardiovasc Surg 2018;51:399-402.

Hata M, Zittermann A, Hakim-Meibodi K, Börgermann J, Gummert J. Minimally invasive mitral valve repair or replacement for degenerative mitral regurgitation. Interact Cardiovasc Thorac Surg 2018;28:575-580.

Kim BS, Soltesz EG, Cohn LH. Minimally invasive approaches to aortic valve surgery: Brigham experience. Semin Thorac Cardiovasc Surg 2006; 18:148-153.

Neves P, Ponce P, Braga P, Vouga L. Minimally invasive aortic coarctation correction and aortic valve replacement without syetnotomy. Rev Port Cir Cardiotorac Vasc 2018;25:127-129.

Reardon MJ, Conklin LD, Philo R, et al. The anatomical aspects of minimally invasive cardiac valve operations. Ann Thorac Surg 1999;67:266-268 